\title{
A New Feature: National Healthcare Safety Network Annual Data
}

\author{
Suzanne F. Bradley, MD
}

Healthcare epidemiologists and infection control practitioners have been under great pressure to rapidly reduce rates of infection due to antimicrobial-resistant pathogens. Government agencies, third-party payers, administrators, and the public have demanded that strategies be implemented to reduce the incidence of these infections. How will we know that these strategies work and are cost-effective? Knowing the baseline infection rate in your facility is a start, and achieving a reduction in that rate is a laudable achievement. However, do you really know how your efforts compare with those of other facilities nationwide?

Voluntary reporting of US infection rate data using standardized definitions and methods began with the National Nosocomial Infections Surveillance system. More recently, the National Healthcare Safety Network (NHSN) was created to expand on this conceptual framework. Findings of surveillance for healthcare-associated infections, including in nonintensive care unit settings, are now reported through the NHSN patient safety component.

In this issue, the journal is pleased to publish updated national antibiotic resistance data provided by the Division of Healthcare Quality and Promotion, of the Centers for Disease Control and Prevention. ${ }^{1}$ For this report, antibiotic-resistant bacteria were chosen for inclusion on the basis of their frequency, clinical importance, and potential concern for emerging resistance. These data were derived from 2 modules of the NHSN patient safety component from January 2006 through October 2007. The modules included data on deviceassociated infections and procedure-associated infections following selected operative procedures. Device-associated infections were central line-associated bloodstream infection, catheter-associated urinary tract infection, and ventilator-associated pneumonia. Postprocedure infections included surgical site infection and postprocedure pneumonia.

As the authors of the report acknowledge, most of the reporting hospitals are large tertiary care centers that provide a wide variety of services to patients with complex conditions and severe illness. However, despite these limitations, rapid provision of timely national data on antimicrobial-resistant pathogens is highly likely to be of great use to our readers as they make decisions regarding the implementation of measures to control infections and the measurement of the effectiveness of these strategies. The editors are hopeful that these annual updates will remain a regular feature of the journal for many years to come.

\section{REFERENCE}

1. Hidron AI, Edwards JR, Patel J, et al. Antimicrobial-resistant pathogens associated with healthcare-associated infections: annual summary of data reported to the National Healthcare Safety Network at the Centers for Disease Control and Prevention, 2006-2007. Infect Control Hosp Epidemiol 2008; 29:996-1011 (in this issue). 